\title{
Measurement of nasal potential difference in young children with an equivocal sweat test following newborn screening for cystic fibrosis
}

\author{
Isabelle Sermet-Gaudelus, ${ }^{1}$ Emmanuelle Girodon, ${ }^{2}$ Delphine Roussel, ${ }^{1}$ Eric Deneuville, ${ }^{3}$ \\ Stéphanie Bui, ${ }^{4}$ Frédéric Huet, ${ }^{5}$ Marcel Guillot, ${ }^{6}$ Rola Aboutaam, ${ }^{1}$ Michel Renouil, ${ }^{7}$ \\ Anne Munck, ${ }^{8}$ Marie des Georges, ${ }^{9}$ Albert Iron, ${ }^{4}$ Christel Thauvin-Robinet, ${ }^{5}$ \\ Isabelle Fajac, ${ }^{10}$ Gerard Lenoir, ${ }^{1}$ Michel Roussey, ${ }^{3}$ Aleksander Edelman ${ }^{1}$
}

\begin{abstract}
- Supplementary material including figures and additional methods are published online only. To view these files please visit the journal online (http:// thorax.bmj.com).
\end{abstract}

${ }^{1} \mathrm{CRCM}$ and INSERM U845, Hôpital Necker, Université René Descartes, Paris, France

${ }^{2}$ Service de Génétique

Moléculaire, Hôpital Henri

Mondor, France

${ }^{3}$ CRCM, Hôpital Pontchaillou,

France

${ }^{4}$ Génétique Médicale et CRCM

Pédiatrique, Hôpital Pellegrin

France

${ }^{5}$ CRCM, Hôpital du Bocage

France

${ }^{6}$ CRCM, Hôpital Robert Bisson

France

${ }^{7}$ CRCM, Hôpital de Saint Pierre, France

${ }^{8}$ CRCM, Hôpital Robert Debré, France

${ }^{9}$ Service de Génétique

Médicale, Hôpital Arnaud de

Villeneuve, France

${ }^{10} \mathrm{CRCM}$, Hôpital Cochin, France

\section{Correspondence to}

Isabelle Sermet-Gaudelus,

CRCM, Hôpital Necker-Enfants Malades and INSERM U845,

Faculté de Médecine Necker,

Université René Descartes, 156

rue de Vaugirard, 75730 Paris,

France;

isabelle.sermet@nck.aphp.fr

Received 11 July 2009

Accepted 2 April 2010

\section{ABSTRACT}

Background A challenging problem arising from cystic fibrosis (CF) newborn screening is the significant number of infants with hypertrypsinaemia (HIRT) with sweat chloride levels in the intermediate range and only one or no identified CF-causing mutations.

Objectives To investigate the diagnostic value for CF of assessing CF transmembrane conductance regulator (CFTR) protein function by measuring nasal potential difference in children with HIRT.

Methods A specially designed protocol was used to assess nasal potential difference (NPD) in 23 young children with HIRT (3 months-4 years) with inconclusive neonatal screening. Results were analysed with a composite score including CFTR-dependent sodium and chloride secretion. Results were correlated with genotype after extensive genetic screening and with clinical phenotype at follow-up 3 years later.

Results NPD was interpretable for 21 children with HIRT: 13 had NPD composite scores in the CF range. All 13 were finally found to carry two CFTR mutations. At follow-up, nine had developed a chronic pulmonary disease consistent with a CF diagnosis. The sweat test could be repeated in nine children, and six had sweat chloride values $\geq 60 \mathrm{mmol} / \mathrm{l}$. Of the eight children with normal NPD scores, only two had two CFTR mutations, both wide-spectrum mutations. None had developed a CF-like lung disease at follow-up. The sweat test could be reassessed in five of these eight children and all had sweat chloride values $<60 \mathrm{mmol} / \mathrm{l}$. CF diagnosis was ruled out in six of these eight children.

Conclusion Evaluation of CFTR function in the nasal epithelium of young children with inconclusive results at CF newborn screening is a useful diagnostic tool for CF.

\section{INTRODUCTION}

Cystic fibrosis (CF) is caused by mutations in the gene encoding the CF transmembrane conductance regulator (CFTR) protein, which acts as a chloride channel after activation by protein kinase A (PKA). ${ }^{1}$ Classically, the diagnosis is based on a characteristic clinical disease with a sweat chloride $\left(\mathrm{Cl}^{-}\right)$ concentration $>60 \mathrm{mmol} / \mathrm{l}$ and/or the identification of two CF-causing mutations. ${ }^{2}$

Newborn screening (NBS) has modified this diagnostic strategy. ${ }^{3}$ This public health programme substitutes preclinical features for later clinical symptoms, thus enabling early management of infants with CF and, accordingly, a better longterm prognosis. ${ }^{4}$ Most CF NBS protocols use immunoreactive trypsinogen (IRT) as the primary screening test and then confirm the CF diagnosis by identifying a CF-causing mutation on each allele or a sweat $\mathrm{Cl}^{-}$concentration $\geq 60 \mathrm{mmol} / 1$, or both. ${ }^{5}$

One important problem arising from CF NBS is that at least $1-2 \%$ of infants with hypertrypsinaemia (HIRT) (ie, IRT above the 99th percentile) have sweat $\mathrm{Cl}^{-}$levels in the intermediate range (eg, between 30 and $59 \mathrm{mmol} / \mathrm{l}$ ) and one or no identified CF-causing mutation. ${ }^{6}$ Some may remain asymptomatic or develop extremely mild phenotypes that would never be noted clinically as part of the CF spectrum. Conversely others will turn out to have symptoms consistent with CF or develop CFTR-related disorders (CFTR-RDs), clinical entities associated with CFTR mutations that do not meet the current diagnostic criteria for CF. The diagnosis may remain inconclusive in these situations when extensive genetic studies identify genetic variations with an unclear pathogenic potential or detect mutations associated with a wide spectrum of phenotypes. ${ }^{7}$ There is thus a need for additional tools beyond the sweat test to sort this dilemma out

In such cases, CFTR function evaluation may serve as a surrogate marker for CF diagnosis. It can be indirectly assessed by measuring nasal transepithelial potential difference (NPD) changes after pharmacological activation of PKA. ${ }^{8}$ CFTR-dependent $\mathrm{Cl}^{-}$secretion is absent in classic CF disease and is normal or only minimally reduced among heterozygotes. ${ }^{9}$ This test provides a valuable diagnostic tool for patients with CF-like symptoms; to distinguish those with non-classic CF and evidence of CFTR dysfunction from those whose normal CFTR function indicates that they are unlikely to have CF. ${ }^{10}$ However, no study has reported the use of NPD for infants with persistently elevated IRT and an inconclusive diagnosis

We adapted our NPD protocol to very young children and conducted a collaborative study in infants with a persistently raised IRT during CF NBS, intermediate sweat chloride concentrations (between 30 and $59 \mathrm{mmol} / \mathrm{l}$ ) and inconclusive genetic findings. To investigate whether the early demonstration of CFTR dysfunction in nasal epithelium is associated with the subsequent appearance of a CF clinical phenotype, NPD results 
were correlated with the children's clinical phenotype at followup 3 years later and with their genotype after extensive genetic screening.

\section{METHODS \\ Population}

Twenty-three children with HIRT were referred from various CF centres in France from January 2006 to December 2008. All had intermediate sweat $\mathrm{Cl}^{-}$concentrations from 30 to $59 \mathrm{mmol} /{ }^{11}$ In accordance with the two-step French NBS strategy, ${ }^{12} 18$ infants were referred to CF centres after birth because of neonatal IRT above the 99th percentile of normal values and one mutation identified from a panel of 33 CF-causing mutations. Five other children had no mutation identified at the first step but their IRT remained elevated at 3 weeks of life. The NeckerEnfants Malades Institutional Review Committee approved the protocol, and each parent provided written informed consent.

These children were compared with the local reference population.

- 29 healthy non-smoking control subjects with no family history of CF, a sweat $\mathrm{Cl}^{-}$concentration $<30 \mathrm{mmol} / 1$ and none of the 30 CFTR mutations most prevalent in the French population

- 67 patients with CF with typical disease, sweat chloride concentration $\geq 60 \mathrm{mmol} / \mathrm{l}$ and CF-causing mutations on both alleles.

\section{Nasal potential difference}

The 'infant' protocol was derived from our previously published standardised protocol for patients older than 5 years. ${ }^{9}$ The test was performed with children lying comfortably in a supine lateral recumbent position. Twenty-one children had light sedation (intrarectal diazepam $0.5 \mathrm{mg} / \mathrm{kg}$ for those younger than 6 months or midazolam $0.3 \mathrm{mg} / \mathrm{kg}$ for older infants). NPD was measured between a reference electrode positioned on a slightly abraded area of the forearm and a measuring electrode linked to the nasal mucosa by a specially designed single-use sterile double-lumen PVC catheter (EU certificate 0337/B5/02, Marquat Génie Médical, Boissy Saint Leger, France).

The basal potential difference (PD) was recorded from the mucocutaneous junction at $2.5 \mathrm{~mm}$ intervals along the lateral margin of the floor to determine the point of maximal baseline PD. Perfusion of the nasal mucosa with Ringer solution was increased according to tolerance from $1 \mathrm{ml} / \mathrm{min}$ to $3 \mathrm{ml} / \mathrm{min}$, instead of the $5 \mathrm{ml} / \mathrm{min}$ rate of the standard protocol. Baseline PD was measured after perfusion of the nasal epithelium with Ringer saline solution, and $\mathrm{PD}$ changes were recorded after perfusion with $100 \mu \mathrm{M}$ amiloride in saline solution to block $\mathrm{Na}^{+}$current (referred to as $\Delta$ Amiloride) and then after $100 \mu \mathrm{M}$ amiloride plus $10 \mu \mathrm{M}$ isoproterenol in $\mathrm{Cl}^{-}$-free solution, to stimulate PKA-dependent CFTR-related $\mathrm{Cl}^{-}$conductance (referred to as $\Delta \mathrm{LowCl}^{-}$-Iso). Solutions were changed as soon as a steady voltage tracing was achieved for at least $30 \mathrm{~s}$, and the differences in NPD values were measured between the plateaus of the corresponding solutions.

Oxygen saturation and cardiac frequency were monitored throughout the test. All tests were performed and interpreted by one investigator, blinded to their clinical and genetic characteristics. The results were not given to the families.

In a preliminary study, the same investigator used both the standard applied routinely at Necker hospital and this 'infant' protocol to take NPD measurements in three healthy adults and three adolescents with classic CF. The tests were conducted on two successive days, with the standard protocol tested first. The infant protocol was subsequently tested on very young children to verify its feasibility.

\section{Genotype analysis}

CFTR genotype data were analysed using a comprehensive mutation analysis. ${ }^{13-16}$

The mutations were classified according to the literature and the European consensus ${ }^{17}$ as: (A) mutations that cause CF disease; (B) mutations that result in a CFTR-RD; (C) mutations with no clinical consequences; or (D) mutations of unproven or uncertain clinical relevance. Mutations associated with a wide phenotypic spectrum that might belong to either group $A$ or group $\mathrm{B}$ were noted as $\mathrm{AB}$.

\section{Clinical assessment}

The presence of phenotypic features consistent with a diagnosis of CF was assessed. ${ }^{2}$ 3 1118 Chronic sinopulmonary disease was defined as: (1) chronic cough and sputum production; and/or (2) colonisation of respiratory tract samples or oropharyngeal swabs by typical CF pathogens including Staphylococcus aureus or Pseudomonas aeruginosa; and/or (3) persistent chest radiograph abnormalities (eg, bronchiectasis, atelectasis, infiltrates or hyperinflation); and/or (4) airway obstruction manifested by wheezing and air trapping; and/or (5) recurrent lower respiratory tract infection defined by at least four episodes per year requiring modification of basal treatment. ${ }^{18}$ Gastrointestinal and nutritional abnormalities included (1) exocrine pancreatic insufficiency $^{19}$; and/or (2) failure to thrive; and/or (3) hepatic CF disease.

\section{Statistics}

Data are presented as means with their SD, or, when not normally distributed, as medians and their IOR. Comparisons between groups were analysed by the Wilcoxon rank sum test or the Mann-Whitney test for quantitative variables and the Fisher exact test for qualitative variables.

Case-control comparisons and discriminant analysis were used to explore which NPD test measurements (alone or in combination) best discriminated all patients with CF from the control population. Standardised beta coefficients were then attributed to each significant variable to form a composite score that provided the best overall discrimination between the two groups. The Fisher linear discriminant function was then applied to determine a cut-off point between the two populations.

\section{RESULTS}

The study enrolled 23 children with HIRT (3 months-4 years). At the time of NPD testing, 14 children were younger than 2 years, including five aged from 3 to 6 months.

\section{Validation of the diagnostic composite NPD score}

A case-control analysis of our CF and the control population allowed us to determine that a $\Delta \mathrm{LowCl}^{-}$-Iso value above $-6 \mathrm{mV}$ was indicative of CF. However, although the specificity of this threshold for CF diagnosis was good (96\%), its sensitivity for diagnosing the disease was insufficient for our purposes (93\%). We therefore used discriminant analysis to develop the following composite score: $-0.11 \Delta \mathrm{LowCl}^{-}$-Iso $-0.05 \Delta$ Amiloride. We determined a diagnostic cut-off of $\leq 0.27$ for $\mathrm{CF}$ that discriminated all patients with CF from all healthy subjects (online supplement). This score was then validated in a second adult population of 88 patients with CF and 33 controls.

\section{Comparison of the infant and the standard paediatric NPD protocols}

In the preliminary study comparing results obtained from the standard and 'infant' protocols in three healthy adults 
(22-38 years) and three adolescents with classic CF (11-18 years), results were similar among both the control subjects and the patients with CF (results shown in the online supplement).

The infant protocol was next applied to three young children, two with CF-causing mutations and one healthy control child. The results were in the same range as those of the corresponding reference $\mathrm{CF}$ and healthy populations (figure 1).

\section{NPD results in the children with HIRT}

Tolerance of the NPD test was good for all patients with HIRT. The NPD results were reliable for 21 of the 23 children. As a group, the patients with HIRT differed significantly from both the patients with CF and the controls for both $\mathrm{Cl}^{-}$secretion and $\mathrm{Na}^{+}$absorption (table 1). The diagnostic score classified patients into two groups: 13 in the CF range (diagnosis score $\leq 0.27$; HIRT-CF) and eight in the control range (diagnosis score $>0.27$, HIRT-Nl). The two groups differed significantly for the response to amiloride and to isoproterenol in low $\mathrm{Cl}^{-}$solution (table 1, figure 2). The patients in the HIRT-Nl group had normal $\mathrm{Na}^{+}$ transport, and their $\Delta \mathrm{LowCl}^{-}$-Iso values, reflecting $\mathrm{Cl}^{-}$secretion, were always above the normal repolarisation threshold of $-6 \mathrm{mV}$. Patients in the HIRT-CF group had NPD results in the CF range with low response to isoproterenol in low chloride solution, and a high response to amiloride.

\section{Characteristics of the patients with HIRT according to CFTR function}

The characteristics of the patients enrolled are shown in the online supplement. At birth, the children did not differ significantly between the two groups for IRT and birth weight (table 2). Four of the HIRT-NI group had a history of neonatal hospitalisation and were born with low birth weight and chronic fetal distress, a clinical condition that has been reported to increase IRT transiently.

At evaluation, the two groups did not differ with regard to pulmonary symptoms (table 2 ). Sweat $\mathrm{Cl}^{-}$concentrations were less elevated in the HIRT-CF group but nevertheless ranged between 30 and $57 \mathrm{mmol} / \mathrm{L}$. After a mean follow-up of 3 years, the HIRT-CF group had a significantly higher rate of CF-like lung disease symptoms ( $n=9$ vs none in the HIRT-Nl group; $\mathrm{p}=0.001$; Fisher exact test). Paeruginos $a$ was isolated only among the HIRT-CF group. Chronic $S$ aureus colonisation was seen in three patients of the HIRT-CF group, whereas patients with HIRT-Nl always had a transient colonisation. Importantly, three patients with HIRT-CF developed bronchiectasis, underscoring the need for an early definitive diagnosis to prevent this irremediable sequela. Two children with a diagnosis score in the $\mathrm{CF}$ range had, respectively, a CFTR-related hepatopathy and a pancreatic insufficiency. Sweat $\mathrm{Cl}^{-}$concentrations were significantly higher in the group with abnormal NPD. Six of them had sweat $\mathrm{Cl}^{-}$levels $\geq 60 \mathrm{mmol} / 1$ compared with none among those with normal NPD.

Altogether, by the 3-year follow-up, 11 of the 13 children in the HIRT-CF group had phenotypic features consistent with CF (either clinical or sweat test results), and none in the HIRT-N1 group ( $p=0.0002$; Fisher exact test). A diagnosis of CF was ruled out in six of the eighr patients with HIRT-Nl but none of the patients with HIRT-CF ( $p=0.0005$; Fisher exact test).

\section{Correlation with the mutation classification}

Of the three patients with no mutation identified, all had normal NPD responses (table 3). Five patients were F508del heterozygous after the initial search for frequent mutations at CF NBS. Three had an abnormal NPD score. A second mutation was subsequently identified in all three $(621+3 \mathrm{~A} \rightarrow \mathrm{G}, \mathrm{R} 933 \mathrm{G}$ and Q1291R) while the other two with diagnostic scores in the normal range had no other mutation and were asymptomatic at follow-up.

All 13 patients in the HIRT-CF group carried two CFTR mutations, compared with two patients in the HIRT-Nl group (Fisher exact test; $\mathrm{p}=0.01$ ): (1) two patients had two CF-causing mutations (A/A) versus none in the HIRT-Nl group; (2) nine patients carried one CF-causing mutation (A) in trans with one

Figure 1 Nasal potential difference (PD) tracings of three infants with cystic fibrosis (CF) and a healthy young child. After securised placement of the catheter at the point of maximal negative voltage on the nasal mucosa, baseline PD is measured after perfusion of the nasal epithelium with saline Ringer solution (S1). PD changes are then recorded after: (1) perfusion with $100 \mu \mathrm{M}$ amiloride in Ringer solution (S2) to block $\mathrm{Na}^{+}$current ( $\triangle$ Amiloride); and (2) $100 \mu \mathrm{M}$ amiloride plus $10 \mu \mathrm{M}$ isoproterenol in chloride-free solution (S3) to stimulate CF transmembrane conductance regulator (CFTR)-related $\mathrm{Cl}^{-}$conductance ( $\Delta \mathrm{LowCl}^{-}$-Iso). The tracings of three infants with $\mathrm{CF}$ (F508del/E1418X, 6 months old; F508del/F508del, 8 months old; and N1303K/N1303K, 18 months old, respectively) are compared with that of a healthy control ( 3 years old, negative neonatal screening, sweat $\mathrm{Cl}^{-}$at $25 \mathrm{mmol} / \mathrm{l}$, no mutation found at

CF Infants

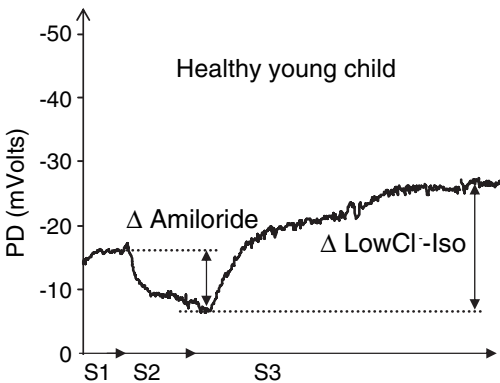

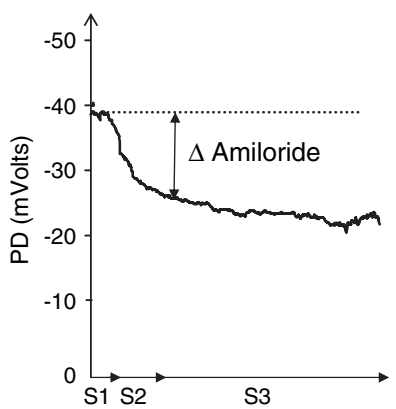

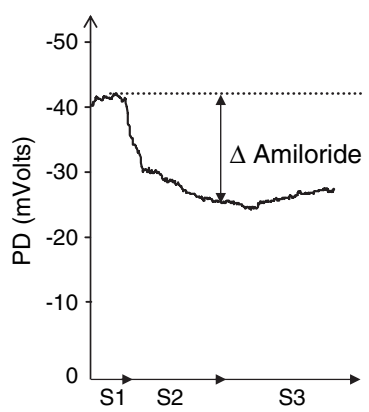

extensive genetic analysis). In the infants with $\mathrm{CF}_{1} \mathrm{Na}^{+}$transport is exaggerated as shown by the elevated basal PD and the increased response to amiloride, and the chloride transport is absent, as shown by the low response to isoproterenol in low chloride solution. 
Table 1 Nasal potential difference (PD) results in infants with hypertrypsinaemia (HIRT) compared with those with classic cystic fibrosis (CF) and healthy controls (Ctrl)

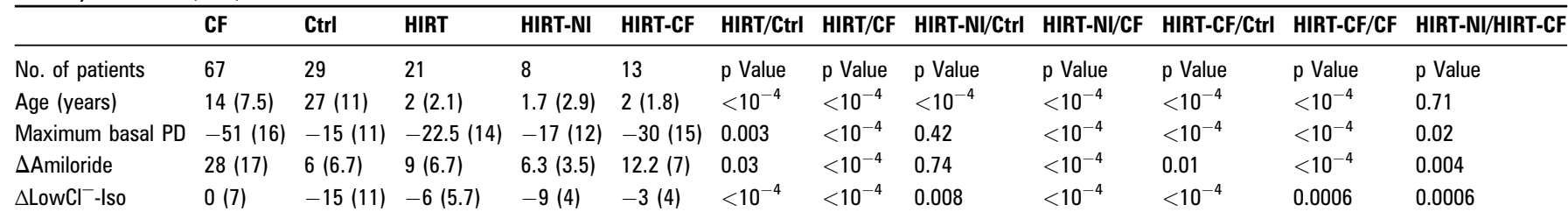

Results are presented as median (IOR). Comparison with Mann-Whitney test.

Patients with HIRT-NI have a diagnostic score $>0.27$, patients with HIRT-CF have a diagnostic score $\leq 0.27$.

broad-spectrum mutation $(A B)$ versus only two patients in the group with a normal score; and (3) two patients had a CFcausing mutation (A) in trans with a sequence variation of unknown clinical relevance $(D)$ versus none in the group with normal CFTR function. Of the three patients with two CFTR mutations in the HIRT-N1 group, one carried a mutation without any clinical consequence $(3849+45 \mathrm{G} \rightarrow \mathrm{A})$, while the other two carried F508del in trans with the R347H broad-spectrum mutation, or the CFTR-RD-associated mutation $\mathrm{R} 117 \mathrm{H}$.

Five patients with HIRT were compound heterozygous for the CF-causing mutations F508del and R117H on an intron 8 T7 background. One displayed a normal PD response and remained asymptomatic at 4 years of age; among the four remaining with an abnormal NPD diagnostic score $<0.27$, two patients had clinical evidence of CF lung disease at follow-up.

\section{DISCUSSION}

This prospective study demonstrates that very young children with HIRT with a borderline sweat test and defective CFTR function in the nasal epithelium are at risk of a worse clinical outcome in later childhood than their counterparts with normal NPD. They are also significantly more likely to carry a CFcausing mutation in trans with another CF- or CFTR-RD-associated mutation.

\section{Limitations of the study}

Despite numerous studies that have sought to assess the role of NPD as a diagnostic tool for CF disease, the test has been neither standardised nor validated for diagnostic accuracy. ${ }^{20}$ We therefore used discriminant function analysis to determine a composite score that best discriminated between CF and healthy subjects. The cut-off point was validated in a second population, so that, according to this rigorous methodology, we can now be confident to apply this score for assessment of CFTR dysfunction.

Little has been reported about NPD in very young children, mostly because of the challenges of performing this test in a population unable to cooperate. ${ }^{21}$ Our methods differed from those in the previous studies by Southern and Gaillard: we used a non-perfusing method (ECG cream) because it provided more stable tracings, a different customised catheter and low chloride solution of slightly different composition. The shortened protocol used in this study yielded results similar to those with the standard protocol in testing three healthy controls and three patients with CF, indicating that (1) the gradient for $\mathrm{Cl}^{-}$ secretion obtained with the decreased flow rate of $3 \mathrm{ml} / \mathrm{min}$ was sufficient to differentiate healthy controls from patients with $\mathrm{CF}$, and (2) the maximal change after isoproterenol in low $\mathrm{Cl}^{-}$ perfusion with the infant protocol was similar to that obtained for the standard protocol in which isoproterenol was added after an initial perfusion with low $\mathrm{Cl}^{-}$solution. ${ }^{22}$

We could not perform a rigorous case-control study including healthy infants and those with CF because of obvious ethical concerns. However, we were able to verify both in a few healthy toddlers and in those with CF older than 6 months of age that the level of $\mathrm{Cl}^{-}$secretion is similar to that in older children, as shown in two previous studies. ${ }^{8} 21$

\section{Interest in NPD assessment for CF diagnosis in very young children}

NPD evaluation, according to the diagnosis score, clearly identified a group of 13 infants with abnormal NPD. Although individual values were more dispersed than in the control group of older patients with two CF-causing mutations, these children had a chloride secretion level approaching that found in typical patients with CF. CFTR function was normal in eight other children.

This diagnostic classification was confirmed at follow-up. Six of the eight patients with normal CFTR function were finally diagnosed with a condition other than CF, and 12 of the 13 patients with HIRT-CF developed either phenotypic features consistent with CF (including chronic productive cough, $P$ aeruginosa, chronic $S$ aureus colonisation, bronchiectasis,
Figure 2 Potential difference (PD) changes after $100 \mu \mathrm{M}$ amiloride in Ringer solution ( $\Delta$ Amiloride) and $10 \mu \mathrm{M}$ in low chloride solution with isoproterenol ( $\Delta \mathrm{LowCl}^{-}$-Iso) in 67 patients with cystic fibrosis (CF), 29 healthy controls (Ctrl), 13 children with hypertrypsinaemia (HIRT) with

a composite diagnosis score $\leq 0.27$ (HIRT-CF) and eight children with HIRT with a composite diagnosis score $>0.27$ (HIRT-NI). The results for the healthy 3year-old child can be seen in the Ctrl group.
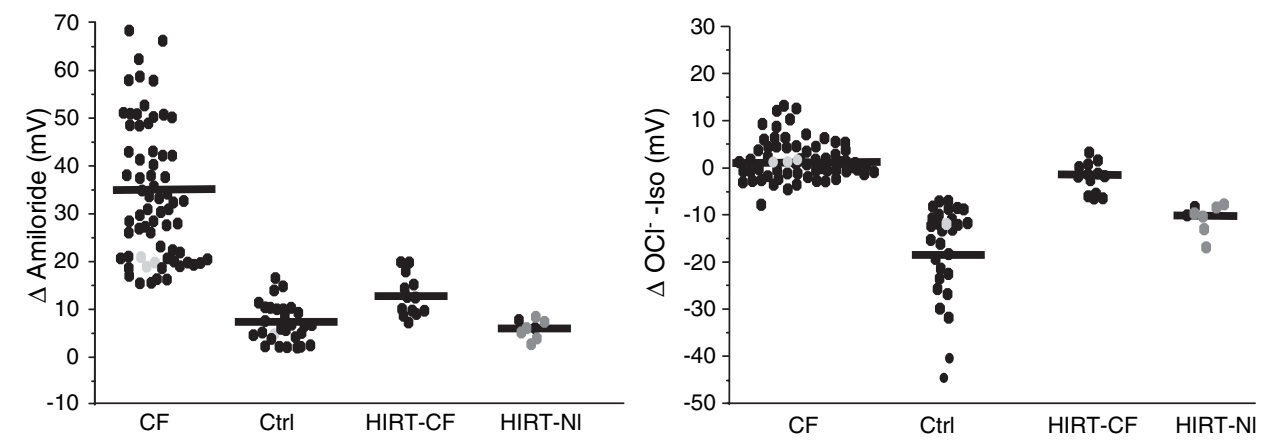
Table 2 Clinical characteristics of children with hypertrypsinaemia (HIRT) at birth and at follow-up according to the diagnostic score cut-off

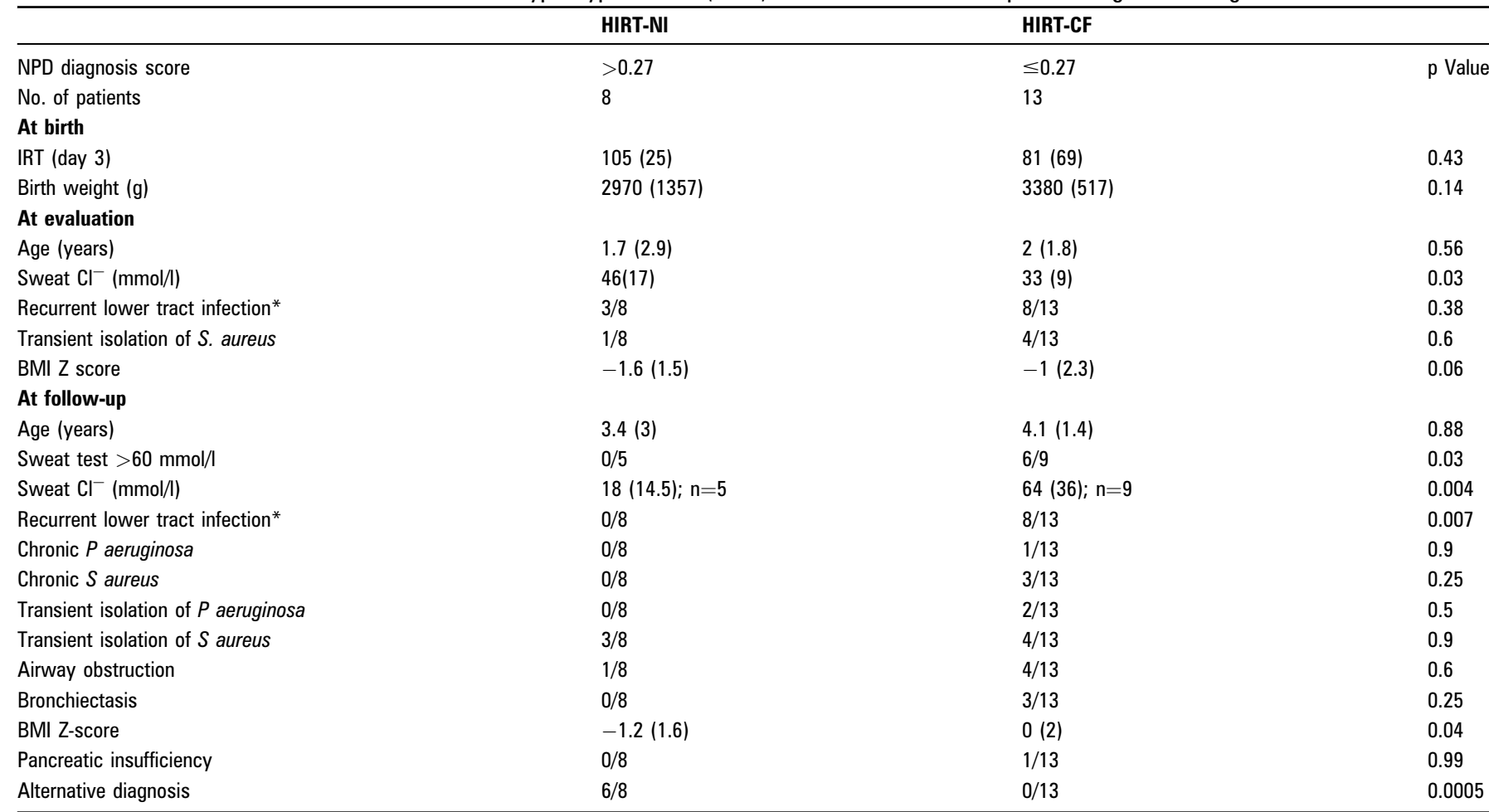

Results are presented as median (IOR) or number of observations. Comparison with Fisher exact test for qualitative variables and Mann-Whitney test for quantitative variables.

Patients with HIRT-NI have a diagnostic score $>0.27$, patients with HIRT-CF have a diagnostic score $<0.27$.

*At least four episodes per week requiring modification of basal treatment.

$\mathrm{BMI}$, body mass index; $\mathrm{CF}$, cystic fibrosis; IRT, immunoreactive trypsinogen; NPD, nasal potential difference.

pancreatic insufficiency) or sweat $\mathrm{Cl}^{-}$concentration levels $>60 \mathrm{mmol} / \mathrm{l}$.

\section{Correlation between NPD assessment and CFTR genotype}

Extensive mutation analysis of the patients with HIRT found that all 13 patients in the HIRT-CF group carried two CFTR mutations, including at least one CF-causing mutation. Of the second alleles, eight were not detected by commercial diagnostic assays. This was, in particular, true for the three HIRT F508del heterozygous patients at initial genetic screening who had

Table 3 Genotypes of the children with HIRT according to the diagnostic score cut-off in the 21 patients with reliable NPD tests; results after extensive genetic analysis

\begin{tabular}{|c|c|c|}
\hline \multirow[b]{2}{*}{ CFTR genotypes } & \multicolumn{2}{|l|}{ Diagnosis score } \\
\hline & $>0.27$ (8 patients) & $\leq 0.27$ (13 patients) \\
\hline $\mathrm{A} / \mathrm{A}$ & 0 & $\begin{array}{l}\text { F508del/ } 621+3 A \rightarrow G \\
\text { F508del/ } 01291 R\end{array}$ \\
\hline$A / A B$ & $\begin{array}{l}\text { F508del/R347H } \\
\text { F508del/R117H;T7 }\end{array}$ & $\begin{array}{l}\text { W846X/R117C } n=2 \\
\text { F508del/R1070W } \\
2183 A A \rightarrow G / L 206 W \\
\text { F508del/3272-26A } \rightarrow G \\
\text { F508del/R117H;T7; } n=4\end{array}$ \\
\hline$A / D$ & 0 & $\begin{array}{l}\text { F508del/R933G } \\
\text { G551D/R3520 }\end{array}$ \\
\hline$B / D$ & $\mathrm{G} 622 \mathrm{D} / 3849+45 \mathrm{G} \rightarrow \mathrm{A}$ & 0 \\
\hline $\mathrm{A} / \mathrm{O}$ & F508del/0 $n=2$ & 0 \\
\hline $0 / 0$ & 3 & 0 \\
\hline
\end{tabular}

0 , no identified mutation; $A$, CF-causing mutation; $B$, mutation associated with cystic CFTRrelated disorders; $C$, mutation with no clinical consequence ; $D$, mutation of unknown or uncertain clinical relevance; $A B$, mutation that is associated with a wide phenotypic spectrum that might belong to either group $A$ or $B$.

CFTR, cystic fibrosis transmembrane conductance regulator; HIRT, hypertrypsinaemia; NPD, nasal potential difference. abnormal NPD scores. These observations suggest that finding an ion transport defect in the nasal epithelium of infants with HIRT who have only one CF-causing mutation at the first stage of the neonatal screening genetic analysis should lead to a more intensive search for a second mutation. Therefore, a two-step diagnostic strategy in children with HIRT with borderline sweat test could include NPD first and be followed by exhaustive genetic screening only if NPD results are in the CF range.

Of the eight patients with normal NPD scores, only two carried two CFTR mutations. Specifically, both had F508del and a wide clinical spectrum mutation associated with normal clinical status in adulthood. These two patients were asymptomatic at follow-up. Conversely, patients with other widespectrum mutations and abnormal NPD scores later turned out to have CF. These findings suggest therefore that the identification of a CF-like functional defect in patients carrying such wide-spectrum mutations might be predictive of a CF-like disease, while normal CFTR function would be associated with, at most, mild non-specific disease.

Five children carrying the F508del/R117H;T7 genotypes were investigated. Two developed early CF-like pulmonary disease, and a third had recurrent wheezing. Interestingly, all three had defective CF-like $\mathrm{Cl}^{-}$secretion. Although we cannot rule out a mutation in a non-coding region or in the promoter region, despite extensive genotyping, these findings suggest that the rare association of CFTR-related clinical symptoms with the F508del/ $\mathrm{R} 117 \mathrm{H} ; \mathrm{T} 7$ genotype may be assessed by NPD measurements. ${ }^{24} 25$

\section{CONCLUSION}

Although the clinical significance of CFTR dysfunction in the newborns with HIRT can only be definitively determined through systematic long-term follow-up, our results suggest 
that assessment of CFTR function in early infancy helps to identify infants at risk of developing a CFTR-related lung disease that requires active follow-up and intense treatment. Conversely, the demonstration of normal CFTR function does not definitively exclude the possibility of mild CFTR dysfunction but appears to rule out typical CF disease with a high degree of probability and should prompt a more thorough investigation for an alternative diagnosis. The avoidance of unnecessary anxiety for families and of useless burdensome treatment for children appears to make this test very valuable in clinical practice. $^{26}$

Funding Supported by Assistance Publique des Hôpitaux de Paris, Vaincre La Mucoviscidose and ABCF Mucoviscidose.

Competing interests None.

Ethics approval This study was conducted with the approval of the Necker-Enfants Malades Institutional Review Committee.

Provenance and peer review Not commissioned; externally peer reviewed.

\section{REFERENCES}

1. Rowe S, Miller S, Sorscher EJ. Cystic fibrosis. N Engl J Med 2005;352:1992-2001.

2. Rosenstein BJ, Cutting GR. for the Cystic Fibrosis Foundation Consensus Panel. The diagnosis of cystic fibrosis: a consensus statement. J Pediatr 1998;132:689-95.

3. Farrell PM, Rosenstein BJ, White TB, et al. Guidelines for diagnosis of cystic fibrosis in newborns through older adults. Cystic Fibrosis Foundation consensus report. J Pediatr 2008:153:S4-S14.

4. Farrell PM, Kosorok MR, Rock MJ, et al. Early diagnosis of cystic fibrosis through neonatal screening prevents severe malnutrition and improves long-term growth. Pediatrics 2001;107:1-13.

5. Southern KW, Munck A, Pollitt R, et al. A survey of newborn screening for cystic fibrosis in Europe. J Cyst Fibr 2007:6:57-65.

6. Parad RB, Comeau AM. Diagnostic dilemmas resulting from the immunoreactive trypsinogen/DNA cystic fibrosis newborn screening algorithm. J Pediatr 2005;147 (3 Suppl):S78-82.

7. Castellani C, Benetazzo MG, Tamanini A, et al. Analysis of the entire coding region of the cystic fibrosis transmembrane regulator gene in neonatal hypertrypsinaemia with normal sweat test. J Med Genet 2001;38:202-5.

8. Knowles MR, Paradiso AM, Boucher RC. In vivo nasal potential difference: techniques and protocols for assessing efficacy of gene transfer in cystic fibrosis. Hum Gene Ther 1993;6:445-55.

9. Sermet-Gaudelus I, Dechaux M, Vallée B, et al. Chloride transport in nasal ciliated cells of cystic fibrosis heterozygotes. Am J Respir Crit Care Med 2005; 171:1026-31.
10. Wilschanski M, Famini H, Strauss-Liviatan N, et al. Nasal potential difference measurements in patients with atypical cystic fibrosis. Eur Respir J 2001;17:1208-15.

11. De Boeck K, Wilschanski M, Castellani C, et al. Cystic fibrosis: terminology and diagnostic algorithms. Thorax 2006;61:627-35.

12. Munck A, Dhondt JL, Sahler C, et al. Implementation of the French nationwide cystic fibrosis newborn screening program. J Pediatr 2008;153:228-33.

13. Fanen $\mathbf{P}$, Ghanem N, Vidaud $\mathrm{M}$, et al. Molecular characterization of cystic fibrosis 16 novel mutations identified by analysis of the whole cystic fibrosis conductance transmembrane regulator (CFTR) coding regions and splice site junctions. Genomics 1992;13:770-6.

14. Le Maréchal C, Audrezet MP, Quéré I, et al. Complete and rapid scanning of the cystic fibrosis tranmembrane conductance regulator (CFTR) gene by denaturing highperformance liquid chromatography (D-HPLC): major implications for genetic counselling. Hum Genet 2001;108:290-8.

15. Audrézet MP, Chen JM, Raguénès 0 , et al. Genomic rearrangements in the CFTR gene: extensive allelic heterogeneity and diverse mutational mechanisms. Hum Mutat 2004:23:343-57.

16. Niel F, Martin J, Dastot-Le Moal F, et al. Rapid detection of CFTR gene rearrangements impacts on genetic counselling in cystic fibrosis. J Med Genet 2004;41:e118.

17. Castellani C. Cuppens $\mathrm{H}$, Macek M Jr, et al. Consensus on the use and interpretation of cystic fibrosis mutation analysis in clinical practice. J Cyst Fibr 2008; 7:179-86.

18. Goubau C, Wilschanski M, Skalická V, et al. Phenotypic characterization of patients with intermediate sweat chloride values: towards validation of the European diagnostic algorithm for cystic fibrosis. Thorax 2009;64:683-91.

19. Walkowiak J, Herzig KH, Strzykala K, et al. Feacal elastase-1 is superior to fecal chymotrypsin in the assessment of pancreatic involvement in cystic fibrosis. Pediatrics 2002;110:e7.

20. Bossuyt P, Reitsma J, Bruns D, et al. Towards complete and accurate reporting of studies of diagnostic accuracy: the STARD initiative. Clin Chem 2003:49:1-6.

21. Southern KW, Noone PG, Bosworth DG, et al. A modified technique for measurement of nasal transepithelial potential difference in infants. $J$ Pediatr 2001;139:353-8.

22. Sermet-Gaudelus I, Roussel D, Bui S, et al. The CF-CIRC study; a French collaborative study to assess the accuracy of cystic fibrosis diagnosis in neonatal screening. BMC Pediatr 2006;6:25-30.

23. Sermet-Gaudelus I, Girodon E, Huet F, et al. Nasal potential difference in cystic fibrosis diagnosis of very young children. J Pediatr 2007;150:e34-5.

24. Thauvin-Robinet C. Munck A. Huet F, et al. The very low penetrance of cystic fibrosis and CFTR-related disorders in individuals with the [R117H;T7]+[F508del] genotype: a reappraisal for genetic counselling and diagnostic practice. J Med Genet 2009;46:752-8.

25. O'Sullivan BP, Zwerdling RG, Dorkin $\mathrm{HL}$, et al. Early pulmonary manifestation of cystic fibrosis in children with the DeltaF508/R117H-7T genotype. Pediatrics 2006:118:1260-5.

26. Mayell SJ, Munck A, Craig JV, et al. A European consensus for the evaluation and management of infants with an equivocal diagnosis following newborn screening for cystic fibrosis. J Cyst Fibr 2008;8:71-8. 